\title{
ОБ АППРОКСИМАЦИИ ФУНКЦИЙ В ГИЛЬБЕРТОВОМ ПРОСТРАНСТВЕ И КУБАТУРНЫХ ФОРМУЛАХ
}

В работе доказываются утверждения, позволяющие для ряда классов функций получать наилучшие кубатурные формулы. В основе рассуждений лежит следующая известная $\left[{ }^{1}\right]$

Т е о р е м а 1. Пусть заданы гильбертово пространство Н со скалярным произведением $(f, \varphi)_{H}$, линейно независимые функции $\pi_{1}, \ldots, \pi_{N} \in H$. Для заданной функции $f \in H$ существует единственный набор чисел $\lambda_{1}, \ldots, \lambda_{N}$ (обозначим их через $\lambda_{1}^{*}, \ldots, \lambda_{N}^{*}$ ), для которых величина

$$
\left\|f-\sum_{k=1}^{N} \lambda_{k} \pi_{k}\right\|_{H}
$$

достигает наименьшего значения (обозначим его через б). Числа $\lambda_{1}^{*}, \ldots, \lambda_{N}^{*}$ являются единственным решением системы

$$
\sum_{k=1}^{N} \lambda_{k}\left(\pi_{k}, \pi_{j}\right)_{H}=\left(f, \pi_{j}\right)_{H} \quad(j=1, \ldots, N)
$$

$u$

$$
\delta^{2}=\|f\|_{H}^{2}-\sum_{k=1}^{N} \lambda_{k}\left(f, \pi_{k}\right)_{H} .
$$

Введем обозначения. Пусть $H_{1}$ - гильбертово пространство функций $\varphi(z)$, рассматриваемых на множестве $z \in E_{1}$, в котором задано скалярное произведение $(\alpha(z), \gamma(z))_{H_{1}} ; H_{2}$ - гильбертово пространство функций $\psi(w)$, определенных на множестве $w \in E_{2}$, со скалярным произведением $(\beta(w), \mu(w))_{H_{2}} ; H_{3}-$ гильбертово пространство функций $f(z, w)$, определенных на множестве $E_{1} \times E_{2}$, со скалярным произведением $\left(f_{1}, f_{2}\right)_{H_{3}}$, удовлетворяющим условию

$$
(\alpha(z) \beta(w), \gamma(z) \mu(w))_{H_{3}}=(\alpha(z), \gamma(z))_{H_{\mathrm{i}}}(\beta(w), \mu(w))_{H_{2}} .
$$

Пусть $m, n, v_{11}, \ldots, v_{1 m}, v_{21}, \ldots, v_{2 n}$ заданы. Функции $K_{1 i}(z, u)$, $K_{2 j}(w, v)(i, j=1,2, \ldots)$ считаем заданными такими, что для любых $u \in E_{1}, v \in E_{2}$ имеем

$$
K_{1 i}(z, u) \in H_{1}, \quad K_{2 j}(w, v) \in H_{2} \quad(i, j=1,2, \ldots),
$$

а векторы $\bar{u}=\left(u_{1}, \ldots, u_{m}\right), \bar{v}=\left(v_{1}, \ldots, v_{n}\right)$ - такими, что множества 


$$
\begin{aligned}
& \left\{K_{11}\left(z, u_{1}\right), \ldots, K_{1 v_{11}}\left(z, u_{1}\right), \ldots, K_{11}\left(z, u_{m}\right), \ldots, K_{1 v_{1 m}}\left(z, u_{m}\right)\right\}, \\
& \left\{K_{21}\left(w, v_{1}\right), \ldots, K_{2 v_{14}}\left(w, v_{1}\right), \ldots, K_{21}\left(w, v_{n}\right), \ldots, K_{2 v_{2 n}}\left(w, v_{n}\right)\right\}
\end{aligned}
$$

состоят из линейно независимых функций.

Через $A_{k i}(\bar{u})$ и $B_{l j}(\bar{v})\left(k=1, \ldots, m ; i=1, \ldots, v_{1 k} ; l=1, \ldots, n\right.$; $\left.j=1, \ldots, v_{2 l}\right)$ обозначим значения $A_{k i}$ и $B_{l j}$, для которых при заданных $\bar{u}$ и $\bar{v}$ величины

$$
\left\|\varphi(z)-\sum_{k=1}^{m} \sum_{i=1}^{v_{1 k}} A_{k i} K_{1 i}\left(z, u_{k}\right)\right\|_{H_{i}}, \quad\left\|\psi(w)-\sum_{l=1}^{n} \sum_{j=1}^{v_{2 l}} B_{l j} K_{2 j}\left(w, v_{l}\right)\right\|_{H_{3}}
$$

достигают наименьших значений (соответственно $\delta_{1}(\bar{u})$ и $\delta_{2}(\bar{v})$ ).

По теореме 1 числа $A_{k i}(\bar{u})$ удовлетворяют равенствам

$$
\begin{array}{r}
\sum_{k=1}^{m} \sum_{i=1}^{v_{1 h}} A_{k i}(\bar{u})\left(K_{1 i}\left(z, u_{k}\right), K_{1 p}\left(z, u_{\lambda}\right)\right)_{H_{1}}=\left(\varphi, K_{1 p}\left(z, u_{\lambda}\right)\right)_{H_{1}} \\
\left(i=1, \ldots, m ; p=1, \ldots, v_{1 \lambda}\right)
\end{array}
$$

и

$$
\delta_{1}^{2}(\bar{u})=\|\varphi\|_{H_{1}}^{2}-\sum_{k=1}^{m} \sum_{i=1}^{v_{1 k}} A_{k i}(\bar{u})\left(\varphi, K_{1 i}\left(z, u_{k}\right)\right)_{H_{i}} ;
$$

а числа $B_{l j}(\bar{v})$ - равенствам

$$
\begin{array}{r}
\sum_{l=1}^{n} \sum_{j=1}^{v_{2 l}} B_{l j}(\bar{v})\left(K_{2 j}\left(w, v_{l}\right), K_{2 q}\left(w, v_{s}\right)\right)_{H_{2}}=\left(\psi, K_{2 q}\left(w, v_{s}\right)\right)_{H_{2}} \\
\left(s=1, \ldots, n ; q=1, \ldots, v_{2 s}\right)
\end{array}
$$

и

$$
\delta_{2}^{2}(\bar{v})=\|\psi\|_{H_{2}}^{2}-\sum_{l=1}^{n} \sum_{j=1}^{v_{2 l}} B_{l j}(\bar{v})\left(\psi, K_{2 j}\left(w, v_{l}\right)\right)_{H_{2}} .
$$

Т е о р ем а 2. Пусть $\varphi \in H_{1}, \psi \in H_{2}, \bar{u}, \bar{v}$ заданы, множества (2) состоят из линейно независимых функций. Тогда величина

$$
\left\|\varphi(z) \psi(w)-\sum_{k=1}^{m} \sum_{l=1}^{n} \sum_{i=1}^{v_{1 k}} \sum_{j=1}^{v_{2 l}} C_{k l}^{(i, j)} K_{1 i}\left(z, u_{k}\right) K_{2 j}\left(w, v_{l}\right)\right\|_{H_{3}}
$$

достигает наименьиего значения при единственных

$$
\left(k=1, \ldots, m ; i=1, \ldots, v_{1 k} ; l=1, \ldots, n ; j=1, \ldots, v_{2 l}\right),
$$

и это значение равно

$$
\mu(\bar{u}, \bar{v})=\left[\|\varphi\|_{H_{1}}^{2}\|\psi\|_{H_{2}}^{2}-\left(\|\varphi\|_{H_{1}}^{2}-\delta_{1}^{2}(\bar{u})\right)\left(\|\psi\|_{H_{2}}^{2}-\delta_{2}^{2}(\bar{v})\right)\right]^{1 / 2} .
$$

Д ок а з а те льст во. По теореме 1 числа $C_{k l}^{(i, j)}$, доставляющие величине (8) наименьшее значение, являются единственным решением системы 


$$
\begin{gathered}
\sum_{k=1}^{m} \sum_{l=1}^{n} \sum_{i=1}^{v_{1 k}} \sum_{j=1}^{v_{2 l}} C_{k l}^{(i, j)}\left(K_{1 i}\left(z, u_{k}\right) K_{2 j}\left(w, v_{l}\right), K_{1 p}\left(z, u_{\lambda}\right) K_{2 q}\left(w, v_{s}\right)\right)_{H_{3}}= \\
=\left(\varphi \psi, K_{1 p}\left(z, u_{\lambda}\right) K_{2 q}\left(w, v_{s}\right)\right)_{H_{3}} \\
\left(\lambda=1, \ldots, m ; p=1, \ldots, v_{1 \lambda} ; s=1, \ldots, n ; q=1, \ldots, v_{2 s}\right) .
\end{gathered}
$$

Если эту систему переписать, учитывая свойство (1), а также равенства (4) и (6), то станет очевидным, что ей удовлетворяют числа (9). По теореме 1 имеем, что

$$
\mu^{2}(\bar{u}, \bar{v})=\|\varphi \psi\|_{H_{3}}^{2}-\sum_{k=1}^{m} \sum_{l=1}^{n} \sum_{i=1}^{v_{1 h}} \sum_{j=1}^{v_{2 l}} A_{k i}(\bar{u}) B_{l j}(\bar{v})\left(\varphi \psi, K_{1 i}\left(z, u_{k}\right) K_{2 j}\left(w, v_{l}\right)\right)_{H_{3}},
$$

откуда по (1), (5) и (7) следует (10), и этим теорема доказана.

Теперь рассмотрим величины $\delta_{1}(\bar{u})$ и $\delta_{2}(\bar{v})$ как функции от $\bar{u}$ и $\bar{v}$. При этом через $\delta_{1}(\bar{u})$ и $\delta_{2}(\bar{v})$ обозначаем наименьшие значения величин (3) при фиксированных $\bar{u}$ и $\bar{v}$ и не требуем линейной независимости функций (2). Пусть $\quad u_{1}^{*}, \ldots, u_{m}^{*} \in E_{1}, \quad v_{1}^{*}, \ldots, v_{n}^{*} \in E_{2}$, $\bar{u}^{*}=\left(u_{1}, \ldots, u_{m}\right), \quad \bar{v}^{*}=\left(v_{i}^{*}, \ldots, v_{n}^{*}\right)$, множества (2) при $\bar{u}=\bar{u}^{*}$, $\bar{v}=\bar{v}^{*}$ состоят из линейно независимых функций и векторы $\bar{u}^{*}$ и $\bar{v}^{*}$ доставляют величинам $\delta_{1}(\bar{u})$ и $\delta_{2}(\bar{v})$ наименьшие значения.

Т е о рем а 3. Числа

$$
\begin{gathered}
u_{k}=\dot{u_{k}}, \quad v_{l}=v_{l}^{*}, C_{k l}^{(i, j)}=A_{k i}\left(\bar{u}^{*}\right) B_{l j}\left(\bar{v}^{*}\right) \\
\left(k=1, \ldots, m ; l=1, \ldots, n ; i=1, \ldots, v_{1 k} ; j=1, \ldots, v_{l l}\right)
\end{gathered}
$$

доставляют величине (8) как функции от $u_{k}, v_{l}, C_{h l}^{(i, j)} \quad(k=1, \ldots, m$; $\left.i=1, \ldots, v_{1 k} ; l=1, \ldots, n ; j=1, \ldots, v_{2 l}\right)$ наименьшее значение, равное $\mu\left(\bar{u}^{*}, \bar{v}^{*}\right)$.

Дока зательство. По определению

$$
0 \leqslant \delta_{1}(\bar{u}) \leqslant\|\varphi\|_{H_{1}}, \quad 0 \leqslant \delta_{2}(\bar{v}) \leqslant\|\psi\|_{H_{2}} .
$$

Поэтому из (10) следует, что величина $\mu(\bar{u}, \bar{v})$ принимает как функщия от $\bar{u}, \bar{v}$ наименьшее значение при $\delta_{1}(\bar{u})=\delta_{1}\left(\bar{u}^{*}\right), \delta_{2}(\bar{v})=\delta_{2}\left(\bar{v}^{*}\right)$. Но тогда по теореме 2 числа $C_{k l}^{(i, j)}$, для которых это значение достигается, равны $A_{k i}\left(\bar{u}^{*}\right) B_{l j}\left(\bar{v}^{*}\right)$, что и доказывает теорему.

Рассмотрим использование этих результатов для построения наилучших кубатурных формул (с прямоугольной сеткой узлов) на заданных классах функций.

Введем в рассмотрение классы функций. Пусть число $M>0$ задано. Через $W\left(r_{t}\right) L_{2}$ обозначим класс всех функций $f(x)$, которые на отрезке $[0,1]$ имеют абсолютно непрерывную производную порядка $r_{i}-1$ и производную порядка $r_{i}$, для которой

$$
\left[\int_{0}^{1}\left|f\left(r_{t}\right)(x)\right|^{2} d x\right]^{1 / 2} \leqslant M
$$

Пусть $L_{i j} y=0\left(j=1, \ldots, r_{i}\right)$ - линейные однородные краевые условия такие, что задача

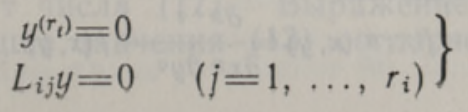


имеет функцию Грина, которую обозначим через $g_{i}(x, t) ; i=1,2$. Через $W\left(r_{t}\right) L_{2}$ обозначим класс всех функций $f(x)$, принадлежащих классу $W^{\left(r_{t}\right)} L_{2}$ и удовлетворяющих условиям $L_{i j} f=0\left(j=1, \ldots, r_{i}\right)$. Частными случаями таких классов являются, например, широко известные классы $\underset{0}{W(r)} L_{2}, \quad W_{01}^{(2 r)} L_{2} \quad\left[{ }^{2}\right]$. Если $f(x) \in \underset{g_{t}}{\left(r_{t}\right)} L_{2}$, то имеет место интегральное представление

$$
f(x)=\int_{0}^{1} f^{\left(r_{t}\right)}(t) g_{i}(x, t) d t .
$$

Квадратурная формула

$$
\int_{0}^{1} p_{i}(x) f(x) d x=\sum_{k=1}^{n_{i}} \sum_{j=0}^{\rho_{i, k}} A_{k j} f^{(j)}\left(x_{k}\right)+R_{n_{i}}(f),
$$

где $p_{i}(x)$ суммируема на $[0,1], \varrho i, 1, \ldots, \varrho_{i, n_{t}}<r_{i}$, называется, согласно С. М. Никольскому $\left[{ }^{2}\right]$, наилучшей на классе $\underset{g_{l}}{\left(r_{t}\right)} L_{2}$, если ее веса и узлы $A_{k j}, x_{k}\left(k=1, \ldots, n_{i} ; j=0, \ldots, \varrho_{i, k}\right)$ выбраны так, что величина

$$
R_{n_{i}}=\sup _{j \in W_{g_{l}}^{\left(r_{i}\right)} L_{2}}\left|R_{n_{i}}(f)\right|
$$

имеет наименьшее значение.

Воспользовавшись известной методикой $\left[{ }^{2}\right]$, легко получим, что

$$
R_{n_{i}}=M d_{i},
$$

где

$$
d_{i}=\left[\int_{0}^{1}\left|\int_{0}^{1} p_{i}(x) g_{i}(x, t) d x-\sum_{k=1}^{n_{i}} \sum_{j=0}^{\rho_{i, k}} A_{k j} \frac{\partial^{j}}{\partial x^{j}} g_{i}\left(x_{k}, t\right)\right|^{2} d t\right]^{1 / 2}
$$

Через $W_{g_{1} g_{2}}^{\left(r_{1}, r_{2}\right)} L_{2}$ обозначим класс всех функций $f(x, y)$, которые на квадрате $0 \leqslant x, y \leqslant 1$ имеют непрерывные производные $f^{(i+j)}(x, y)$ $\left(i<r_{1}, j<r_{2}\right)$, кусочно непрерывные производные $f^{\left(r_{1}+j\right)}(x, y), f^{\left(i+r_{2}\right)}(x, y)$ $\left(j=0, \ldots, r_{2} ; i=0, \ldots, r_{1}\right)$, удовлетворяют по переменной $x$ краевым условиям

$$
L_{1 j} f \equiv 0 \quad\left(j=1, \ldots, r_{1}\right),
$$

по переменной $y$ - краевым условиям

$$
L_{2 j} f \equiv 0 \quad\left(j=1, \ldots, r_{2}\right)
$$

и неравенству

$$
\left[\int_{0}^{1} \int_{0}^{1}\left|f^{\left(r_{1}+r_{2}\right)}(x, y)\right|^{2} d x d y\right]^{1 / 2} \leqslant M^{*}
$$

Функции этого класса допускают представление

$$
f(x, y)=\int_{0}^{1} \int_{0}^{1} f^{\left(r_{1}+r_{2}\right)}(t, v) g_{1}(x, t) g_{2}(y, v) d t d v .
$$

* Мы пользуемся обозначением

$$
f^{(p+q)}(x, y)=\frac{\partial^{p+q}}{\partial x^{p} \partial y^{q}} f(x, y) .
$$


Кубатурная формула

$\int_{0}^{1} \int_{0}^{1} p_{1}(x) p_{2}(y) f(x, y) d x d y=\sum_{k=1}^{n_{1}} \sum_{l=1}^{n_{2}} \sum_{i=0}^{\rho_{1, k}} \sum_{j=0}^{\rho_{2, l}} A_{k l}^{(i, j)} f^{(i+j)}\left(x_{k}, y_{l}\right)+R_{n_{1} n_{2}}(f)$

называется наилучшей на классе $W_{g_{1} g_{2}}^{\left(r_{1}, r_{2}\right)} L_{2}$, если ее узлы $\left(x_{k}, y_{l}\right)$ и веса $A_{k l}^{(i, j)}$ выбраны так, что величина

$$
R_{n_{1} n_{2}}=\sup _{f \in W_{g_{1} g_{2}}^{\left(r_{1}, r_{2}\right)} L_{2}}\left|R_{n_{1} n_{2}}(f)\right|
$$

принимает наименьшее значение.

Подставляя (14) в (15), получаем, как и в одномерном случае, что

$$
R_{n_{1} n_{2}}=M d
$$

где

$$
\begin{gathered}
d=\left[\iint_{0}^{1} \int_{0}^{1} \mid \int_{0}^{1} \int_{0}^{1} p_{1}(x) p_{2}(y) g_{1}(x, t) g_{2}(y, v) d x d y-\right. \\
\left.-\left.\sum_{k=1}^{n_{1}} \sum_{l=1}^{n_{2}} \sum_{i=0}^{\rho_{1, k}} \sum_{j=0}^{\rho_{2, l}} A_{k l}^{(i, j)} \frac{\partial^{i}}{\partial x^{i}} g_{1}\left(x_{k}, t\right) \frac{\partial^{j}}{\partial y^{j}} g_{2}\left(y_{l}, v\right)\right|^{2} d t d v\right]^{1 / 2}
\end{gathered}
$$

Следовательно, задача построения наилучшей формулы (15) на $W_{g_{1} g_{2}}^{\left(r_{1}, r_{2}\right)} L_{2}$ сводится к минимизации величины (16) по узлам и весам, что можно сделать с помощью теоремы 3.

Пусть для каждого $i=1,2$ на классе $W_{g_{t}}^{\left(r_{t}\right)} L_{2}$ наилучшая формула (12) имеет узлы $x_{i, k}\left(k=1, \ldots, n_{i}\right)$ (различные и принадлежащие отрезку $[0,1])$, веса $A_{k j}^{(i)} \quad\left(k=1, \ldots, n_{i} ; j=0, \ldots, \varrho_{i, k}\right)$ и оценку остатка $R_{n_{i}}=M \bar{d}_{i}$.

Обозначим

$$
\varphi_{i}=\left[\int_{0}^{1}\left|\int_{0}^{1} p_{i}(x) g_{i}(x, t) d x\right|^{2} d t\right]^{1 / 2}
$$

Теорем а 4. Наилучшая на классе $W_{g_{1} g_{2}}^{\left(r_{1}, r_{2}\right)} L_{2} \quad$ кубатурная формула (15) имеет узлы и веса

$$
\begin{gathered}
x_{k}=x_{1, k} ; \quad y_{l}=x_{2, l} ; \quad A_{k l}^{(i, j)}=A_{k i}^{(1)} A_{l j}^{(2)} \\
\left(k=1, \ldots, n_{1} ; i=0, \ldots, Q_{1, h} ; l=1, \ldots, n_{2} ; j=0, \ldots, \varrho_{2, l}\right)
\end{gathered}
$$

и оценку остатка

$$
R_{n_{1} n_{2}}=M\left[\varphi_{1}^{2} \varphi_{2}^{2}-\left(\varphi_{1}^{2}-\bar{d}_{1}^{2}\right)\left(\varphi_{2}^{2}-\bar{d}_{2}^{2}\right)\right]^{1 / 2} .
$$

Доказ ательст в о получается сразу, если заметить, что к величине (16) применима теорема 3 , по которой наименьшее значение этой величине доставляют числа (17). Выражение наименьшего значения (16) через наименьшие значения (13) согласно теореме 3 приводит к значению (18). 
3 а меч ание. Доказанная теорема позволяет сразу выписать наилучшую кубатурную формулу на классе $W_{g_{1} g_{2}}^{\left(r_{1}, r_{2}\right)} L_{2}$, если известны наилучшие квадратурные формулы на классах $W_{g_{t}}^{\left(r_{t}\right)} L_{2}$. Аналоги этой теоремы были получены в $\left[{ }^{3,4}\right]$. Из этой теоремы как частные случаи следуют некоторые ранее полученные результаты (напр., [5,4]). Теорема 4 является следствием теоремы 3. Последняя может быть успешно использована и при построении наилучших кубатурных формул на классах функций, не подчиненных краевым условиям (см., напр., [ $\left.{ }^{6}\right]$ ).

Доказательство теоремы 3 не изменится, если в (8) (и, естественно, в (3)) некоторые узлы считать фиксированными (напр., $u_{1}=1-u_{m}=$ $=v_{1}=1-v_{n}=0$ при $\left.E_{1}=E_{2}=[0,1]\right)$. Но тогда при некоторых фиксированных узлах сохраняет силу и теорема 4, и, следовательно, с ее помощью можно получить и такие результаты, как, например, результаты работ $\left[{ }^{7,8}\right]$.

Рассмотрим в виде примера один частный случай теоремы 4. Пусть $W_{01}^{(2)} L_{2}$ означает класс функций $f(x)$, которые на $[0,1]$ имеют абсолютно непрерывную производную и удовлетворяют условиям $f(0)=f(1)=0,\left[\int_{0}^{1}\left|f^{\prime \prime}(x)\right|^{2} d x\right]^{1 / 2} \leqslant M$.

Пусть $W_{01}^{(2,2)} L_{2}$ означает класс функций $f(x, y)$, которые имеют кусочно непрерывные производные $f_{x^{\prime} y^{\prime}}^{(i+j)}(x, y) \quad(i, j=0,1,2)$ на квадрате $0 \leqslant x, y \leqslant 1$ и удовлетворяют условиям

$$
\begin{gathered}
{\left[\int_{0}^{1} \int_{0}^{1}\left|f_{x^{2} y^{2}}^{(4)}(x, y)\right|^{2} d x d y\right]^{1 / 2} \leqslant M,} \\
f(0, y) \equiv f(1, y) \equiv f(x, 0) \equiv f(x, 1) \equiv 0 .
\end{gathered}
$$

По теореме 4 для того, чтобы получить наилучшую кубатурную формулу на $W_{01}^{(2,2)} L_{2}$, надо повторно применить наилучшую квадратурную формулу на $W_{01}^{(2)} L_{2}$. Воспользовавшись результатом работы $\left[{ }^{9}\right]$, получим следующее утверждение: на классе $\mathbb{W}_{01}^{(2,2)} L_{2}$ наилучшая формула

$$
\int_{0}^{1} \int_{0}^{1} f(x, y) d x d y=\sum_{k=1}^{m} \sum_{l=1}^{n} A_{k l} f\left(x_{k}, y_{l}\right)+R_{m n}(f)
$$

имеет узль

$$
\begin{gathered}
x_{k}=2 h\left(\sqrt{\frac{2}{3}}+k-1\right), \quad y_{l}=2 g\left(\sqrt{\frac{2}{3}}+l-1\right) \\
(k=1, \ldots, m ; \quad l=1, \ldots, n),
\end{gathered}
$$

вeca

$$
\begin{aligned}
& A_{k l}=4 h g, \\
& A_{1 l}=A_{m l}=A_{k 1}=A_{k n}=2 g h\left(\frac{5}{4} \sqrt{\left.\frac{2}{3}+1\right)}\right. \\
& \quad(k=2, \ldots, m-1 ; l=2, \ldots, n-1),
\end{aligned}
$$




$$
A_{11}=A_{1 n}=A_{m 1}=A_{m n}=g h\left(\frac{5}{4} \sqrt{\frac{2}{3}+1}\right)^{2}
$$

и оценку остатка

$$
\sup _{f \in W_{01}^{(2,2)} L_{2}}\left|R_{m n}(f)\right|=\frac{M}{60} \sqrt{\frac{2}{3}\left(h^{4}+g^{4}\right)-\frac{16}{9} h^{4} g^{4} .}
$$

Здесь

$$
h=0,5\left(2 \sqrt{\frac{2}{3}+m-1}\right)^{-1}, \quad g=0,5\left(2 \sqrt{\frac{2}{3}-n-1}\right)^{-1} .
$$

Отметим, что теоремы $2-4$ легко переносятся и на функции произвольного числа переменных.

\section{ЛИТ Е Р А Т Р А}

1. Ахиез ер Н. И., Лекции по теории аппроксимашии, М., 1965.

2. Н и кольски й С. М.. Квадратурные формулы. М., 1974.

3. Л е в и н М., Изв. АН ЭССР, Физ. Матем., 19, 499 (1970).

4. Л е в и М.. Тр. Таллинск. политехн. ин-та, Матем. и теор. мех., VI, 75 (1971).

5. ІШ а ॥ Э., Изв. АН БССР, Физ. Матем., № 3, 68 (1970).

6. Com a n Gh., Stud. Univ. Babes-Bolyai. Ser. math.-mech., 18, 41 (1973).

7. C o m a n Gh., M i c l a Gh., Rend. mat., 4, 303 (1971).

8. Л уш п а й Н. Е., Сб. работ асп. ДГУ (матем. и мех.), Днепропетровск, 1972, с. 35.

9. Л е в и М., Изв. АН ЭССР, Физ. Матем., 18, 249 (1969).
Таллинский политехнический институт Поступила в редакцию
2/III 1976

\section{LEVIN}

\section{FUNKTSIOONIDE APROKSIMEERIMISEST HILBERTI RUUMIS JA KUBATUURVALEMITEST}

Kordajad $C_{h l}^{(i, j)}$ ja sõlmed $\left(u_{k}, v_{l}\right)$ on valitud nii, et suuruse (8) (tingimusel (1)) väärtus oleks minimaalne.

Defineeritakse funktsioonide $f(x, y)$ hulk, mis rahuldab tingimusi

$$
\left\|f^{\left(r_{1}+r_{2}\right)}(x, y)\right\|_{L_{2}[0,1 ; 0,1]} \leqslant M \quad \text { ja } \quad(14) .
$$

Sellele hulgale on leitud parim valem (15), s. t. valemi (15) kordajad ja sõlmed on valitud nii, et $R_{n_{1} n_{2}}$ omaks vähimat väärtust.

\section{LEVIN}

\section{ON THE APPROXIMATION IN HILBERT SPACE AND CUBATURE FORMULAE}

The coefficients $C_{k l}^{(i, j)}$ and knots $\left(u_{k}, v_{l}\right)$ are chosen to minimize the value (8). It is shown that the solution of this problem can be obtained (under condition (1)) by minimizing the values (3).

Let us consider classes of functions $f(x, y)$ satisfying the conditions (14) and

$$
\left\|f\left(r_{1}+r_{2}\right)(x, y)\right\|_{L_{2}[0,1 ; 0,1]} \leqslant M \text {. }
$$

The best formula (15) is found for these classes. In other words, the coefficients and knots of formula (15) are chosen to minimize the value $R_{n_{1} n_{2}}$. 